\title{
Comentarios
}

\section{El conflicto del Estado y las petroleras y los límites del Estado y del mercado}

Desde la liberalización del mercado de productos derivados del petróleo, a principios de septiembre de 1992, las empresas importadoras y distribuidoras han venido operando con una relativa ausencia de regulaciones estatales. Sólo existe una fórmula para calcular los precios de importación de hidrocarburos, conocida como sistema de paridad de precios de importación. En la actualidad, y como resultado del alza en los precios internacionales del petróleo, tanto el gobierno como la mayoría de diputados de la Asamblea Legislativa coinciden en la necesidad de establecer regulaciones a las ganancias de las empresas dedicadas a la importación de hidrocarburos.

Desde el primer momento, quedó claro que la liberalización del mercado de hidrocarburos no concluciría a una situación hipotética de "competencia perfecta" o de eficiencia, debido a que el mercado ha estado dominado casi desde siempre por un oligopolio, integrado por no más de cuatro empresas transnacionales: Esso, Shell, Texaco y Chevron. Hasta 1992, el gobierno se reservaba el derecho de importación de los hidrocarburos con lo cual, además de obtener considerables utilidades, también ejercía alguna regulación de los márgenes de intermediación, cobrados a las distribuidoras y al consumidor. La liberalización del mercado supuso, por una parte, que el Estado saliera del riegocio de los hidrocarburos ( $y$ perdiera las utilidades que anteriormente obtenía) y, por la otra, que los márgenes de intermediación se fijaran a criterio de las empresas dedicadas a la importación, transporte y distribución de hidrocarburos (en la actualidad, limitadas a la Esso, Shell y Texaco).

Coincidentemente, la tendencia de los precios en esos momentos fue hacia el alza, aunque ello no fue suficiente para causar mayores escozores ni en los consumidores ni en el gobierno. Fue hasta la reciente escalada de los precios internacionales del petróleo, solamente comparable a la crisis del petróleo de la década de 1970, que comenzó a considerarse la necesidad de introducir nuevas regulaciones en el mercado. En concreto, la propuesta consiste en regular el margen de intermediación o utilidad bruta obtenido de las petroleras en la importación y el transporte de los hidrocarburos lo cual, obviamente, desató la oposición de aquéllas y forzó un proceso de negociación que, al final, obligó al gobierno a ceder parcialmente respecto a su postura original.

Curiosamente, este conflicto-negociación lo iniciaron los funcionarios del tercer gobierno de Arena, el mismo partido al que pertenecen los gobiernos que dispusieron otorgar contratos muy favorables a las empresas privadas que han adquirido empresas estatales y los que también legislaron para que las tarifas de servicios básicos pudieran ser ajustadas periódicamente para evitar posibles reducciones en las utilidades (como en el caso de la telefonía y la electricidad). Considérese, por ejemplo, el conflicto entre la Superintendencia General de Electricidad y Telecomunicaciones (Siget) y la empresa Nejapa Power. El contrato de suministro de electricidad generada por medios térmicos, suscrito por el gobierno con esa empresa, co- 
loca al Estado en una posición desventajosa ya que, para no incumplir dicho contrato, aquél debe comprar la energía eléctrica a un precio muy elevado, al grado de que el Estado (y los consumidores) al final está obligado a pagar un sobreprecio de 25 millones de dólares anuales por un período de quince años. Inexplicablemente, esta situación se mantuvo durante cinco años, hasta la llegada del tercer gobierno de Arena.

En el caso del reciente conflicto del Estado salvadoreño con las petroleras, vale la pena rescatar el tema de los límites del Estado y del mercado, revisando los principales argumentos que han aflorado, para luego hacer una valoración sobre el impacto de la regulación estatal de las ganancias y sobre otras opciones que pudieran mitigar el alza en los precios de los hidrocarburos.

\section{El conflicto del Estado con las petroleras}

Al examinar los detonantes de este conflicto no puede obviarse la existencia de un doble componente: por una parte, el fuerte incremento de los precios internacionales del petróleo y, por la otra, los relativamente elevados márgenes de intermediación, cobrados por las petroleras a los distribuidores. Curiosamente, ha sido el primer componente el que ha llamado la atención sobre las posibles utilidades extraordinarias que las empresas petroleras estarían obteniendo en El Salvador. Sin duda, ello obedece a que los precios de los productos derivados del petróleo son clave para el funcionamiento de la economía, pues de su comportamiento dependen muchas actividades económicas, que los utilizan como insumo. Así, incrementos en los precios de los combustibles pueden provocar incrementos en los costos y en los precios de las empresas, reducciones en las tasas de crecimiento económico e, inclusive, pueden conducir a situaciones de recesión económica, combinada con desequilibrios del sector externo. Es por ello que el gobierno se interesó por buscar opciones para mitigar el efecto inflacionario del alza en los precios internacionales del petróleo.

Estos últimos son fijados de acuerdo a las políticas adoptadas por la Organización de Países Exportadores de Petróleo (Opep), la cual se encarga de fijar cuotas de producción a sus miembros, de manera que el precio internacional se mantenga dentro de niveles que garanticen la rentabilidad de la extracción y comercialización del crudo. En otras palabras, se encarga de distorsionar el libre mercado. En el momento presente, las políticas adoptadas por la Opep han llegado a ser tan restrictivas como para provocar incrementos considerables en los precios internacionales del barril de petróleo, tal como ha sucedido en los últimos dieciocho meses, en los cuales ha pasado de 18 dólares a más de 32 dólares, lo cual supone un incremento de 77.8 por ciento, aproximadamente.

Fue en este escenario que las petroleras decidieron reducir voluntaria, aunque temporalmente, sus márgenes de ganancia, a partir del 10 de julio: a la gasolina especial se le rebajaron 70 centavos, a la regular 90 centavos y al diesel 40 centavos. Dos meses después se conoció que el gobierno salvadoreño negociaba con las petroleras un acuerdo con nuevas reducciones de sus márgenes de intermediación y, con ello, reducciones en los precios pagados por los consumidores. En concreto, los funcionarios del Ministerio de Economía insistían en que los márgenes podían reducirse todavía más, hasta fijarlos en niveles de 1.12 colones para la gasolina especial, 1.00 colón para la regular y 0.83 centavos para el diesel.

En un primer momento, la discusión se desarrolló entre el Ministerio de Economía y las respectivas gerencias de las tres empresas transnacionales presentes en el país, aunque sin mayores resultados. Debido a lo anterior y al debate público que este tema estaba generando, los diputados de la Comisión de Economía de la Asamblea Legislativa citaron al Ministro de Economía para que explicara el resultado de sus gestiones. En esta reunión, este último dio a conocer que los márgenes de intermediación cobrados por las petroleras en El Salvador casi triplicaban los precios vigentes en Guatemala y Honduras. Así, por ejemplo, si por cada galón de gasolina especial las petroleras de Guatemala y Honduras recibían un margen de 80 y 84 centavos de colón respectivamente, en El Salvador recibían 2.55 colones.

A partir de ello, la Comisión emitió un dictamen en el cual recomendaba que la Asamblea Legislativa aprobara un decreto (conocido luego como Decreto 107) mediante el cual se redujeran los márgenes de intermediación de las empresas petroleras y se fijaran según los montos propuestos por el Ministerio de Economía. Este decreto fue aprobado hasta por los diputados del Partido Arena, principal impulsor de las medidas de liberalización del mercado de los hidrocarburos. 
Lógicamente, la medida generó rápidas y encontradas reacciones de parte de los representantes de las empresas transnacionales y hasta de la Asociación Nacional de la Empresa Privada (Anep). Las primeras señalaron no estar de acuerdo con esta regulación estatal, porque representa un cambio de las "reglas del juego" y porque ellos siguen "las reglas del libre mercado". Esta postura ha sido apoyada también por la Asociación Nacional de la Empresa Privada (Anep) para la cual la intervención del Estado en el mercado de los combustibles no se justifica. De acuerdo a las petroleras, las diferencias entre los márgenes de intermediación cobrados en Guatemala y Honduras se deben a la existencia de diferentes condiciones de mercado: en El Salvador no hay pucrtos en el Atlántico, por lo cual el flete se incrementa, los costos de la mano de obra son mayores, los costos de los terrenos también, el tamaño de mercado es menor, etc.

En la práctica, el Decreto 107 solo sirvió para forzar las negociaciones, que ya de antemano se sostenían, pues desde el principio el presidente Flores condicionó la promulgación del citado decreto a la actitud que asumieran aquéllas, e inclusive se llegó a plantear que el decreto podía ser vetado o modificado, si las compañías disminuían voluntariamente sus márgenes de intermediación. Al final, se llegó a un arreglo en el cual, prácticamente, el gobierno cedió la mayor parte: aceptó una reducción de sólo una tercera parte de la fijada por el decreto legislativo ( 0.21 centavos) y a cambio de ello modificó el mencionado Decreto 107 para ajustarlo a esos montos. Aun así, el Ministro de Economía afirma haberse anotado un triunfo en su empeño por reducir el impacto del alza de los precios internacionales del petróleo sobre los consumidores.

\section{Impacto y opciones}

La reducción final de los márgenes de intermediación es insignificante frente a la escalada de precios de los combustibles. Sólo en las dos semanas siguientes al acuerdo, los precios de la gasolina especial se incrementaron en 1.43 colones, es decir, casi siete veces la reducción negociada por el gobierno.

Aunque se hubiera aprobado el Decreto 107, las rebajas que podrían haberse esperado en el precio final de los combustibles habrían oscilado apenas entre un 2 y un 3 por ciento, lo cual es poco significativo y sugiere que todavía es necesaria la

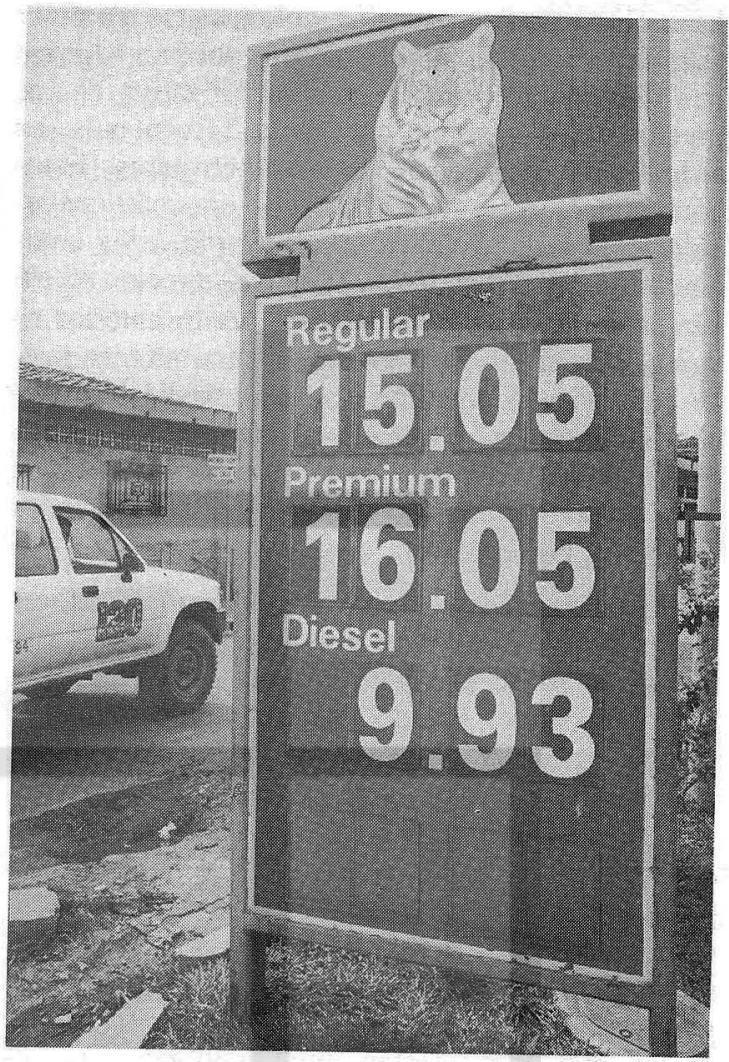

búsqueda de otras alternativas, aunque sin despreciar la regulación de los márgenes de intermediación de las petroleras.

El margen de intermediación de las petroleras es solamente uno de los componentes del precio final de los combustibles, pues también se cuentan el Impuesto al Valor Agregado (Iva), los subsidios al diesel y al gas propano, el margen de las gasolineras y el precio internacional. De éstos solamente el último no puede ser afectado por la política económica, pues los demás sí son susceptibles y, además, pueden ofrecer mejores opciones para mitigar el alza en los precios de los combustibles.

De hecho, la misma Esso Standard Oil señalaba en una carta abierta que "debido a los altos precios se están recaudando más impuestos de lo esperado", específicamente de Iva y en concepto de subsidios al diesel y al gas propano. De éstos, el impuesto total al valor agregado que se aplica a los combustibles ha subido en cerca de 187 por ciento, en los últimos veinte meses. Esto quiere decir, por ejemplo, que el impuesto al valor agregado aplicado a cada galón de gasolina especial se 
ha incrementado en casi 4 colones. De eliminar este excedente, sin duda que el gobiemo lograría una disminución mucho más signifícativa en los precios de la gasolina que la de 21 centavos tan arduamente negociada con las petroleras. Estas contraatacaron, argumentado que era más conveniente "la reducción temporal del Iva a los combustibles, ya que debido a los altos precios se están recaudando más impuestos". A lo anterior se agrega que el margen de la gasolinera también puede ser revisado, mientras que el subsidio al diesel para el transporte público y el gas propano podría obtenerse a través de impuestos menos regresivos (como mayor impuesto sobre la renta de grandes empresas, por ejemplo).

Al margen de lo anterior, es justo reconocer que el conflicto entre el gobierno y las petroleras ha puesto sobre el tapete el tema de la necesaria intervención del Estado para regular condiciones anómalas, como, por ejemplo, la constitución de monopolios u oligopolios, que provocan distorsiones elevando de modo excesivo los precios y o creando escasez "artificial" de un determinado producto. El que las petroleras aceptaran la reducción de 21 centavos y que, además, ya anteriormente hubieran reducido voluntariamente sus márgenes a partir del 10 de julio pasado refleja que, en este mercado, se están obteniendo ganancias innecesariamente altas a costa de los consumidores, quienes se ven obligados a pagar un precio mayor del que se fijaría en un mercado competitivo:

\section{Conclusiones}

El mercado de hidrocarburos es uno de los casos donde se acepta la intervención del Estado para corregir las distorsiones provocadas por el dominio de una o unas pocas empresas. De modo que ni siquiera los máximos apologistas del libre mercado se han escandalizado de la intervención del Estado en el mercado de los productos derivados del petróleo, puesto que lo que se busca es, precisamente, garantizar los beneficios que se generan con la eficiencia atribuida al libre mercado en la asignación de recursos escasos.

En sintonía con lo anterior, el gobierno ha anunciado que promoverá la entrada al mercado de más oferentes, los cuales serían independientes de las compañías petroleras transnacionales e introducirían mayor competencia y eficiencia en el mercado. Para ello, el gobierno inclusive estaría contemplando invertir $\mathbf{4 0}$ millones de colones en la construcción de una nueva planta de almacenamiento de derivados del petróleo.

Los argumentos presentados por las petroleras para justificar sus relativamente altos márgenes de ganancia pueden ser válidos pero, aun así, resulta claro que la rentabilidad obtenida por cada galón de combustible vendido en El Salvador es mayor que la obtenida en Guatemala y Honduras, y así lo refleja el mismo hecho que, aun y cuando se hubiera acatado la reducción por decreto, propuesta por la Asamblea Legislativa, los márgenes de El Salvador todavía estarían cerca de un 30 por ciento por arriba de los cobrados en Guatemala o Honduras, lo cual es más que suficiente para cubrir los supuestos mayores costos que las petroleras enfrentan en El Salvador.

El mercado de hidrocarburos es sólo una pequeña muestra de lo que sucede en El Salvador, donde predominan los mercados monopólicos y oligopólicos. Estos mercados, al igual que en el caso de las petroleras, se creen con derecho a mantener sus privilegios, en aras de la vigencia del "libre mercado". Sin embargo, el conflicto con las petroleras no ha conducido a reflexionar de manera más amplia sobre las implicaciones que está teniendo la entrada de la empresa privada en actividades anteriormente controladas por el Estado (telefonía, generación de energía eléctrica por medios térmicos y distribución de la misma, importación, transporte y distribución de hidrocarburos); pero la verdad es que, al examinar el dilema de los límites del Estado y del mercado, es interesante tener en cuenta no solamente el caso de los hidrocarburos, sino también el de los servicios básicos y el del sistema de pensiones, por ejemplo.

La polémica entre la Superintendencia General de Electricidad y Telecomunicaciones y la empresa generadora de energía Nejapa Power o el alza de las tarifas de energía eléctrica y de las telecomunicaciones, experimentadas desde que entraron en operación las empresas privadas que ahora se benefician de la prestación de este servicio, es una manifestación del mismo problema. Tanto en el caso de las telecomunicaciones como en el de la distribución de energía eléctrica se ha permitido la introducción de cargos fijos y de metodologías para calcular las tarifas que dejan lugar a dudas sobre su idoneidad y que, en la práctica, han significado alzas considerables en el precio de estos servicios. Por otra parte, en el sistema de pensiones ( $y$ en la banca privada) se evidencian cada vez 
con mayor claridad tendencias hacia la fusión de administradoras de los fondos de pensiones y de los bancos, lo cual genera mayor concentración y conduce a la conformación de oligopolios.

Aunque se puede disentir sobre los efectos de la negociación del Estado con las petroleras -pues existen otros componentes del precio de la gasolina que pueden revisarse-, no debe obviarse que es urgente la promulgación de una ley de libre competencia así como también las reformas a la Ley de Protección al Consumidor, de tal manera que se pueda garantizar al menos un mínimo de orden en los procesos de "liberalización económica" tan en boga hoy en día.
A nadie escapa que la inversión extranjera es importante para promover el desarrollo, sin embargo, cuando ésta persigue obtener beneficios extraordinarios de un país, el Estado se encuentra en la obligación de intervenir para corregir las distorsiones del mercado que, a final de cuentas, afectan a los sectores mayoritarios del país, en beneficio de grandes empresas transnacionales. La inversión extranjera no es un fin en sí misma, sino solamente un medio para promover el crecimiento económico y el empleo.

\section{Luis Ernesto Romano}

